\title{
Mentalización en estudiantes universitarios de psicología
}

\author{
Mentalization in psychology college students
}

\author{
Andrea Rodríguez Quiroga* \\ Saskia Ivana Aufenacker** \\ Laura Bongiardino*** \\ Natalia Soracco $* * * *$ \\ Javiera Ortega $* * * * *$ \\ Kevin Scavone****** \\ Natalia Vázquez*******
}

\section{Resumen}

Se considera a la mentalización como una competencia clínica enriquecedora y de utilidad en la formación de psicólogos, especialmente clínicos. El objetivo fue analizar la capacidad de mentalización en los estudiantes de la carrera de psicología e identificar si existían diferencias significativas según el año de cursada de la carrera - $\quad$ primer, tercer y quinto año. La muestra fue de 169 estudiantes universitarios que respondieron a los instrumentos: Mentalization Scale (Ments) y Mentalization Questionnaire (MZQ). Los resultados del estudio demostraron diferencias significativas según el grado de avance de la carrera, en dos dimensiones de la mentalización, la equivalencia psíquica y la regulación del afecto. Es decir, se observa que los estudiantes que están más avanzados en la carrera, obtuvieron mejores niveles de

*Asociación Psicoanalítica de Buenos Aires. Instituto Universitario de Salud Mental ; Pontificia Universidad Católica Argentina. Mail de contacto: andrearodriguezquiroga@ gmail.com

**Asociación Psicoanalítica de Buenos Aires. Instituto Universitario de Salud Mental ; Universidad del Salvador.

*** Asociación Psicoanalítica de Buenos Aires. Instituto Universitario de Salud Mental ; Universidad del Salvador.

**** Pontificia Universidad Católica Argentina.

***** Pontificia Universidad Católica Argentina ; Consejo Nacional de Investigaciones

Científicas y Técnicas.

****** Pontificia Universidad Católica Argentina.

******* Pontificia Universidad Católica Argentina.

DOI: https://doi.org/10.46553/RPSI.17.34.2021.p62-82

Fecha de recepción: 3 de diciembre de 2020 - Fecha de Aceptación: 21 de octubre de 2021 
mentalización, en 2 de las 7 dimensiones evaluadas de la mentalización. Si bien los resultados deben ser considerados con relación a las limitaciones de las escalas. Conclusiones: Vale la pena recalcar que los resultados en relación con la dimensión otros no obtuvieron diferencias significativas a lo largo de la carrera. Dado que el tratamiento psicoterapéutico y su efectividad están ligados a la cualidad de las relaciones con el otro, es importante considerar la posibilidad de su desarrollo durante la formación universitaria y en los inicios de la profesión. El presente estudio representa un aporte significativo, ya que permite plantear la posibilidad del entrenamiento en mentalización como parte de la formación de grado en psicología.

Palabras clave: mentalización, estudiantes universitarios, formación, psicología

\begin{abstract}
Mentalization isconsidered as an enriching and useful clinical competence in the training of psychologists, especially clinical ones. The objective was to analyze the mentalization capacity in psychology students and to identify if there were significant differences according to in which year of study they are in -first, third and fifth year. The sample consisted of 169 university students who responded to the instruments: Mentalization Scale (Ments) and Mentalization Questionnaire (MZQ). The results of the study showed significant differences according to the degree of advancement of the career in two dimensions of mentalization, psychic equivalence and the regulation of affect. That is, it is observed that the students who
\end{abstract}

are more advanced in the career obtained better levels of mentalization, in 2 of the 7 evaluated dimensions of mentalization, although the results must be considered in relation to the limitations of the scales. Conclusions: It is worth emphasizing that the results in relation to the other dimension did not obtain significant differences throughout the career. Given that psychotherapeutic treatment and its effectiveness are linked to the quality of relationships with the other, it is important to consider the possibility of its development during university training and at the beginning of the profession. The present study represents a significant contribution, since it allows raising the possibility of training in mentalization as part of undergraduate training in psychology Key words: mentalization, college students, training, psychology

\section{Introducción}

La tarea clínica de los profesionales de la salud siempre se desarrolla en el marco de una relación humana. La cualidad que dicho vínculo adquiera va a tener un impacto significativo en el resultado terapéutico. Lambert (2013) estimó que la relación terapéutica y las expectativas del paciente son responsables en un $45 \%$ de la mejoría del paciente. Un indicador privilegiado de la relación terapéutica es la alianza terapéutica o alianza de trabajo, cuya calidad puede entenderse como un índice del proceso colaborativo entre paciente y terapeuta. De hecho, Horvath et al. (2011) realizaron un metanálisis que da cuenta de la relación positiva entre la calidad de la alianza terapéutica y los resultados de tratamiento, según diferentes modalidades de tratamiento 
psicológico. La magnitud de esta correlación, junto con los efectos del terapeuta en los procesos terapéuticos, son según Wampold (2001), uno de los más fuertes predictores de éxito en el tratamiento. Stiles et al. ya en 1998, alertaban acerca de la importancia de la respuesta efectiva del terapeuta. La misma es entendida, como la capacidad de éste para darse cuenta y modificar su intervención según los cambios contextuales, incluyendo la percepción de las características de cada uno, o sea paciente y terapeuta, así como sus conductas (Stiles et al.,1998).

El presente artículo hace foco sobre la figura del terapeuta y más específicamente en su formación. Se considera la singularidad del profesional, como un instrumento ejecutor, en tanto es un determinante de las intervenciones sobre la persona del paciente al que asiste.

El Collaborative Research Program on the Development of Psychotherapists (Orlinsky et al., 1999), en un estudio realizado a más de 5000 psicoterapeutas, explicita diferentes modos de involucramiento personal en el tratamiento. El estudio describe: (a) una modalidad afirmativa con uso de estrategias de afrontamiento constructivas y (b) dificultades frecuentes en la práctica, acompañadas por estrategias defensivas, sentimientos de aburrimiento y ansiedad. Se aclara que ambas modalidades son experimentadas en algún grado por todos los terapeutas.

Lo expresado hasta aquí indica que, para cumplir efectivamente la función profesional, no sólo se requieren sólidos conocimientos teóricos y técnicos, sino que además son necesarias ciertas habilidades personales, para facilitar la integración de los aspectos académicos, cognitivos, emocionales e interpersonales.

Ahora bien, ¿cómo es posible saber si un terapeuta está bien entrenado? La investigación en psicoterapia informa que los profesionales del campo de la salud mental no suelen ser evaluados con relación a su efectividad (Lambert, 2013). Es sabido que el terapeuta es responsable de alguna variabilidad en los procesos y resultados de los tratamientos psicoterapéuticos (TP, aproximadamente un $5 \%$ ), aunque aún no hay evidencia suficiente acerca de cuál es su aporte. Es necesario indagar entre otras cuestiones, por ejemplo, que hace que los terapeutas trabajen mejor con algunos pacientes que con otros.

Lambert (2013) plantea algunos desafíos pendientes en relación con esta temática, explicitando que debemos conocer más acerca de: (a) los componentes eficaces de la formación y la supervisión, (b) la interacción entre la formación, la supervisión, la terapia personal, la educación didáctica, las relaciones familiares y de amistad y los eventos externos, (c) cómo y cuánto aprenden los terapeutas de sus experiencias con los pacientes y (d) examinar cómo se incorporan las habilidades con el tiempo. También señala algunos problemas metodológicos a considerar como, por ejemplo, que las formaciones suelen carecer de enfoques definidos, la falta de asignación aleatoria a los sujetos investigados, la falta de atención al abandono de estudiantes y la falta de seguimiento de los estudiantes a posteriori de la formación. A estos problemas se debe sumar el divorcio entre los conceptos teóricos y las técnicas psicoterapéuticas.

Desde esta perspectiva, resulta fundamental que la formación universitaria incluya un entrenamiento en actitudes, 
habilidades y destrezas, que considere a la persona total del profesional de la salud, integrando las dimensiones biopsicosociales. Wampold, ya en el 2001, alertaba respecto de la importancia del entrenamiento de terapeutas con relación al desarrollo de la alianza, entendiendo esta formación como un aspecto de importancia para la práctica clínica. Los terapeutas resaltan que en su formación la experiencia de la práctica clínica es lo que más los ha ayudado.

La formación profesional que los estudiantes deben desarrollar ha sido descripta como competencias del terapeuta en The Competency Benchmarks for Professional Psychology (Fouad et al., 2009). Las mismas han sido definidas por Epstein y Hundert (2002) como: El uso habitual y juicioso de la comunicación, el conocimiento, las habilidades técnicas, el razonamiento clínico, las emociones, los valores y la reflexión en la práctica diaria para el beneficio de la persona y de la comunidad a la que se sirve (p. 226).

Las competencias son dieciséis, agrupadas en 6 bloques. Los puntos de referencia a los que estos bloques aluden son: profesionalismo, relacionamiento, ciencia, aplicación, formación y sistemas. Estos puntos de referencia están diseñados para ser utilizados de forma flexible, indicando tanto expectativas de competencia como formas de calificación que representan los objetivos y los resultados de aprendizaje y que pueden adaptarse al programa de formación particular que se esté implementando.

Es de destacar, con relación a los objetivos de este artículo, la competencia e importancia respecto a relacionarse de manera efectiva y significativa con individuos, grupos $\mathrm{y} / \mathrm{o}$ comunidades.
Se propone que la mentalización sea desarrollada en el transcurso de la formación de grado, desde sus diversas condiciones: como una capacidad humana, como herramienta para comprender al otro y por su relevancia en el rol profesional asistencial (Swan \& Riley, 2015). En este sentido, Freda y colaboradores (2015) señalan la necesidad de promover la mentalización en ámbitos académicos, reafirmando su valor como competencia básica del profesional clínico para enfrentar realidades complejas.

Se define a la mentalización, como la "capacidad exclusivamente humana de interpretar el significado del comportamiento de los demás, considerando sus estados mentales e intenciones subyacentes, así como la capacidad de entender la repercusión que los propios estados afectivos y comportamientos tienen sobre los demás" (Fonagy \& Target, 1996; 2000; Target \& Fonagy, 1996, citados en Midley et al., 2019, p.39).

El concepto de mentalización, propuesto por Fonagy y Target (1996; 2000; 2002; 2006), integra los aportes de maestros del psicoanálisis como Freud, Klein, Bion, Winnicott, Marty y Luket, para luego poner en escena su aporte a la teoría del Apego desarrollada por Bowlby, que proporciona un marco descriptivo y explicativo para entender las relaciones interpersonales entre los seres humanos (1989) y comprender también la transmisión transgeneracional de la subjetividad humana (Fonagy, 2015). Dicha teoría se basa en una formulación interdisciplinaria, que integra campos diversos cómo la evolución por selección natural, la teoría de las relaciones de objeto —psicoanálisis-, la biología evolutiva y los campos de la etología y la psicología 
cognitiva.

La mentalización es una capacidad dinámica que fluctúa, según cada individuo y cada situación. Es susceptible de modificarse por situaciones de estrés y en situaciones donde se activan modelos de apego. También es multifacética, está conformada por cuatro dimensiones: (a) Automática o Controlada (b) Enfocada interna o Externamente (c) Orientada al Self o a otros y (d) Cognitiva o Afectiva. Cada persona puede tener mayor habilidad en algunas dimensiones que en otras, o tender a alguno de los polos de alguna dimensión (Bateman \& Fonagy, 2019).

La ausencia de una mentalización funcional puede llevar a modos de mentalización primitivos. Estos modos primitivos son denominados: equivalencia psíquica, pensamiento teleológico y modo como si — pretend mode en inglés-. La equivalencia psíquica tiene como característica que el individuo vive lo interno como realidad, lo que uno siente y piensa es verdad. Los individuos orientados a un modo teleológico tienden a necesitar pruebas del mundo externo o de otros. Finalmente, el modo como sí acontece cuando los pensamientos y sentimientos se alejan de la realidad. Fallas en los modos de mentalizar pueden ser generados tanto por vivencias traumáticas, fallas en la regulación afectiva, comprensión frágil interpersonal como también, por factores genéticos.

La capacidad del terapeuta para ser consciente de sus propios estados mentales y los de su paciente es considerada una base importante para las técnicas terapéuticas y para la eficacia clínica (Ensink et al., 2013). En la actualidad, este modelo ha adquirido carácter transteórico, considerando a la mentalización como un factor común asociado a la eficacia clínica, independiente de la corriente teórica del profesional.

El tratamiento basado en la mentalización se ha desarrollado en diferentes ámbitos de la práctica clínica y en aplicaciones específicas, entre ellas, el trabajo con niños y adolescentes, con padres y con familias. También diferentes estudios han demostrado que intervenciones basadas en la mentalización son útiles en ámbitos académicos/escolares. La terapia basada en la mentalización para niños (MBT-C) se enfoca en promover explícitamente la mentalización y la reparación de la mentalización cuando se ha perdido (Midgley et al., 2019)

Aunque aún se necesita más investigación en relación a la eficacia de los enfoques de mentalización de la depresión y sus mecanismos de cambio, se cuenta con evidencia acerca de la efectividad del enfoque mentalizador en el tratamiento del trastorno depresivo mayor (Bressi et al., 2017, Jakobsen et al., 2014, Lemma y Fonagy, 2013, Lemma et al., 2011) y en pacientes con trastorno límite de la personalidad (Bateman y Fonagy, 2008; Rossouw y Fonagy, 2012, en Luyten et al., 2019). Estudios recientes demuestran de qué manera la mentalización protege contra la aparición de trastornos borderline de la personalidad en adolescentes (Sharp, 2019). Esto describe algunos de los ámbitos en que la mentalización se ha desarrollado en los últimos 20 años.

La interacción terapéutica tiene como característica esencial que el terapeuta estimule el proceso de mentalización; favoreciendo que el paciente pueda volverse, poco a poco, cada vez más curioso de su propia mente y la de los demás (Bateman \& Fonagy, 2013, 2016). Se plantea que paciente 
y terapeuta están juntos compartiendo un mismo bote; se necesitan dos para poder mentalizar terapéuticamente (Allen, 2003). El terapeuta, en el contexto de una relación de apego con el paciente, emocionalmente cercana, va a permitirle al paciente relacionarse de manera más adaptativa y flexible con los estados emocionales dolorosos (Ward et al., 2009).

Por lo tanto, para fomentar la mentalización del paciente, el terapeuta debe tener buena capacidad de mentalización (Allen, 2003). En este sentido surge el interrogante itienen los terapeutas habilidades de mentalización suficientes como para poder favorecer el proceso de mentalización en sus pacientes? Tener en cuenta la mente del otro y la relación con el otro, son las condiciones sine qua non para promover la mentalización; a la vez, el terapeuta debe estar capacitado para dirigir procesos reflexivos sobre el yo formativo (Fonagy \& Target, 1997; Freda et al., 2015).

Argentina es uno de los países con más psicólogos a nivel mundial. Se estima que en la actualidad hay más de 98.000 psicólogos trabajando activamente y la gran mayoría de ellos lo hace en el ámbito de la atención clínica. Es importante mencionar que, en Argentina, cuando un alumno culmina sus 5 años de carrera recibe el título de grado que es habilitante para el ejercicio profesional, es decir, para la atención clínica de pacientes. Un estudio reciente advierte que no existe un sistema de regulación de la formación apropiado y sostenido para terapeutas. La educación y capacitación de los terapeutas es un desafío futuro, que requiere de un debate y reflexión colectiva (Fierro et al., 2018).

Distintos autores sugieren que la competencia clínica de mentalización es necesaria para la formación de estudiantes de los últimos años o terapeutas noveles, porque implica la posibilidad de tener un pensamiento reflexivo sobre las emociones que surgen en las relaciones (Freda et al., 2015; Goodman, 2013). Una investigación con estudiantes de maestría en psicología clínica demostró que, a partir de un entrenamiento práctico en mentalización, los estudiantes utilizaban más palabras relacionadas a la mentalización (Freda et al., 2015). Otro estudio también demostró mejoras pequeñas pero significativas en la capacidad de mentalización de los terapeutas recientemente graduados, a partir de un breve entrenamiento focalizado en dicha capacidad (Ensink et al., 2013). Esposito et al. (2020) exponen que es posible diseñar intervenciones en contextos universitarios para desarrollar la capacidad de mentalización en estudiantes, lo cual además repercute e incide en el incremento de su bienestar psicológico y de sus logros académicos.

En este sentido, el entrenamiento en mentalización resultaría ser altamente beneficioso por su utilidad transteórica y transdiagnóstica. Sin embargo, los antecedentes son escasos. Se necesitan estudios que pongan a prueba la implementación y posterior evaluación de la eficacia de programas de entrenamiento en mentalización en estudiantes.

Considerando la ausencia de antecedentes a nivel local, se propuso un estudio inicial con el objetivo de evaluar la capacidad de mentalización en estudiantes de distintos años de la carrera de psicología en la Universidad Católica Argentina, sede Buenos Aires. 
Las preguntas de investigación que guiaron el estudio fueron: ¿Cómo son los niveles de mentalización de los estudiantes de la carrera de Psicología? ¿La capacidad de mentalización aumenta según se avanza en la carrera de Psicología?

\section{Metodología}

Se realizó un estudio de diseño no experimental, transversal y correlacional. El objetivo fue analizar la capacidad de mentalización en los estudiantes de la carrera de psicología e identificar si existían diferencias significativas según el año de cursada de la carrera (primer, tercer y quinto año).

\section{Muestra}

La muestra total fue de 169 estudiantes universitarios de psicología de la Pontificia Universidad Católica Argentina, sede Buenos Aires. La edad promedio fue de 20,81 ( $D E$ $=3,45$ ), de los cuales $88,1 \%$ eran mujeres y $11,9 \%$ hombres. En cuanto a la distribución por años de carrera, $37 \%$ eran alumnos del primer año, 30,4\% de tercer año, 30,4\% de quinto año.

\section{Instrumentos}

El protocolo administrado consistió en un consentimiento informado, un cuestionario de datos sociodemográficos y los instrumentos Mentalization Scale y Mentalization Questionnaire (ver Anexo).

La escala Mentalization Scale [MentS] (Dimitrijevic et al., 2018), es una escala autoadministrada que mide la mentalización. La escala se compone de 28 ítems en los que el sujeto debe responder en una escala Likert señalando el nivel de acuerdo o desacuerdo con cada afirmación. Esta escala mide: la mentalización del self, MentS-Self; la mentalización de otros, MentS-Otros y la motivación para mentalizar, MentS-M. Algunos ítems son inversos, por ende, necesitan ser recodificados; estos son los ítems: 8, 9, 11, 14, 18, 19, 21, 22, 26 y 27. De este modo, a mayor puntaje, mayor nivel de mentalización. En cuanto a las propiedades psicométricas originales de la escala, la misma ha demostrado tener validez de constructo, a partir de un análisis factorial de componentes principales y análisis paralelo demostraron la existencia de tres factores que explicaron el $37,51 \%$ de la varianza $(X 2[378]=3778.43, p<0.001$; $\mathrm{KMO}=0.84)$. A su vez, demostró niveles de confiabilidad aceptables en población general $(\alpha=0.75)$ y en muestras clínicas ( $\alpha=0.75$; Dimitrijevic et al., 2018). Los valores de confiabilidad fueron calculados para la muestra de este estudio $(\alpha=0.82)$, presentando valores buenos de acuerdo con el coeficiente alfa de Cronbach (George \& Mallery, 2001).

El Mentalization Questionnaire [MZQ] (Hausberg et al., 2012), es un instrumento autoadministrado, que evalúa la capacidad de mentalización. Este cuestionario está compuesto por 15 ítems divididos en 4 subescalas: Negación de autorreflexión, Conciencia emocional, Modo de equivalencia psíquica y Regulación afectiva. Todos los ítems deben ser recodificados. De este modo, un mayor puntaje implica un mayor nivel de mentalización. En su estudio original, este instrumento ha demostrado validez de criterio a través del método de grupos contrastados y buenos índices de confiabilidad ( $\alpha=0.81$; Hausberg et al., 2012). Para la muestra del presente estudio, se encontraron valores de 
$\alpha=0.76$ para la confiabilidad, aceptables de acuerdo con el coeficiente alfa de Cronbach (George \& Mallery, 2001).

Para la traducción de las escalas de MentS y MZQ. Dos investigadoras bilingües realizaron una traducción de las escalas de inglés al castellano de forma separada. Luego, de estas dos versiones se realizó una versión consensuada en castellano. Esta versión fue supervisada y luego comparada con la versión original en inglés, por la directora de la investigación y la investigadora principal.

\section{Procedimientos}

Cumpliendo con las normas éticas que guían el trabajo de investigación, sólo participaron estudiantes mayores de 18 años que dieron su consentimiento. Se garantizó el anonimato de los datos a través de la codificación de los casos. Todos los sujetos fueron previamente informados de la naturaleza del estudio, participando de manera voluntaria y respetando las directrices éticas de la Declaración de Helsinki (Asociación Médica Mundial, 2017) en todos sus términos. Este estudio fue realizado en el marco del Programa de Acreditación de Proyectos de Investigación (PROAPI) en la Universidad Católica Argentina, sede Buenos Aires.

Se procedió para la recolección de datos mediante cuestionarios impresos de manera presencial, así como también con cuestionarios en línea (Google Forms). Toda la muestra se recabó en el mismo año calendario, 2019.

\section{Análisis de los datos}

El análisis estadístico de los datos se realizó a través del Statistical Package for the Social Sciences (SPSS v.25.0). Se calcularon las medias y desvíos estándar para el análisis descriptivo de las escalas MentS, MZQ y sus dimensiones. Se revisó la distribución de los datos mediante la prueba Shapiro-Wilk y al no tener una distribución normal, se eligió el estadístico Kruskal-Wallis para comparar los grupos de estudiantes de los diferentes años de la carrera de psicología.

\section{Resultados}

\section{Análisis descriptivo de la variable mentalización y sus dimensiones}

Se estudiaron los niveles de mentalización en alumnos de la carrera de psicología a partir de dos instrumentos de auto reporte. A través del instrumento MentS, se midieron las dimensiones motivación hacia la mentalización, mentalización de los otros y mentalización del self.

En cuanto a la dimensión del self, la media mayor se obtuvo en el tercer año de estudio $(M=30,2 ; D E=4,18)$, seguido de quinto año $(M=29,98 ; D E=6,12)$ y primer año $(M=28,75 ; D E=6,1)$. En la dimensión otros tanto primer año $(M=42,4 ; D E=4,36)$ como quinto año $(M=42,22 ; D E=3,95)$ obtuvieron puntuaciones similares y una diferencia de un punto con respecto a tercer año $(M=41,17 ; D E=4,9)$. Por último, en la dimensión de motivación el puntaje más alto se reportó en primer año $(M=42,42 ; D E$ $=2,93)$, luego quinto año $(M=41,9 ; D E=$ $2,98)$ y por último tercer año $(M=40,8 ; D E$ $=3,61)$. Los resultados descriptivos de esta escala y los grupos se muestran en la Tabla 1 .

El análisis descriptivo de la mentalización a través del instrumento MZQ, encontró para la escala total una media de $3,61(D E=0,59)$ para la muestra total.

Es importante considerar como está descripto en materiales, que, a mayor valor en 
70 Mentalización en estudiantes universitarios de psicología

los puntajes obtenidos con este instrumento, el nivel de mentalización es mayor.

En la dimensión de rechazo de la función reflexiva primer y tercer año mantuvieron el mismo puntaje medio 4,01 ( $D E=0,69 ; D E=0,57$ respectivamente) y quinto año obtuvo puntaje medio de 4,17 $(D E=0,65)$. En la dimensión equivalencia psíquica el puntaje mayor lo obtuvo quinto año $(M=3,45 ; \mathrm{DE}=0,87)$ luego tercer año
$(M=3,12 ; D E=0,71)$ y finalmente primer año $(M=3,00 ; D E=1,00)$. En la dimensión regulación afectiva la media mayor se observó en quinto año, luego tercero y por último primero $(M=3,78 ; D E=0,84 ; M$ $=3,71 ; D E=0,75 ; \mathrm{M}=3,33 ; D E=0,85$ respectivamente).

El análisis descriptivo de las subescalas y puntuaciones para cada grupo se muestra en la Tabla 2.

\section{Tabla 1}

Estadísticos descriptivos de mentalización según MentS

\begin{tabular}{|c|c|c|c|c|c|}
\hline Subescalas & Años de estudio & Media & Desvío Estándar & Min & Max \\
\hline \multirow[t]{4}{*}{ Self } & 1 año & 28,75 & 6,1 & 14 & 39 \\
\hline & 3 año & 30,2 & 4,18 & 17 & 40 \\
\hline & 5 año & 29,98 & 6,12 & 14 & 40 \\
\hline & Total & 29,57 & 5,73 & 14 & 40 \\
\hline \multirow[t]{4}{*}{ Otros } & 1 año & 42,4 & 4,36 & 33 & 50 \\
\hline & 3 año & 41,17 & 4,9 & 26 & 49 \\
\hline & 5 año & 42,22 & 3,95 & 28 & 50 \\
\hline & Total & 41,96 & 4,41 & 26 & 50 \\
\hline \multirow[t]{4}{*}{ Motivación } & 1 año & 42,42 & 2,93 & 34 & 49 \\
\hline & 3 año & 40,8 & 3,61 & 29 & 46 \\
\hline & 5 año & 41,9 & 2,98 & 34 & 48 \\
\hline & Total & 41,76 & 3,21 & 29 & 49 \\
\hline
\end{tabular}


Tabla 2

Estadísticos descriptivos de mentalización según MZQ

\begin{tabular}{|c|c|c|c|c|c|}
\hline Subescalas & Año de estudio & Media & Desvío Estándar & Min. & Max. \\
\hline \multirow{4}{*}{$\begin{array}{l}\text { Rechazo de la } \\
\text { función reflexiva }\end{array}$} & 1 año & 4,01 & 0,69 & 2,25 & 5,00 \\
\hline & 3 año & 4,01 & 0,57 & 2,00 & 5,00 \\
\hline & 5 año & 4,17 & 0,65 & 2,75 & 5,00 \\
\hline & Total & 4,06 & 0,64 & 2,00 & 5,00 \\
\hline \multirow{4}{*}{$\begin{array}{l}\text { Equivalencia } \\
\text { psíquica }\end{array}$} & 1 año & 3,00 & 1,00 & 1,25 & 5,00 \\
\hline & 3 año & 3,12 & 0,71 & 2,00 & 4,25 \\
\hline & 5 año & 3,45 & 0,87 & 1,50 & 5,00 \\
\hline & Total & 3,17 & 0,89 & 1,25 & 5,00 \\
\hline \multirow{4}{*}{$\begin{array}{l}\text { Regulación } \\
\text { afectiva }\end{array}$} & 1 año & 3,33 & 0,85 & 1,67 & 5,00 \\
\hline & 3 año & 3,71 & 0,75 & 1,67 & 5,00 \\
\hline & 5 año & 3,78 & 0,84 & 2,00 & 5,00 \\
\hline & Total & 3,58 & 0,83 & 1,67 & 5,00 \\
\hline \multirow[t]{4}{*}{ Total } & 1 año & 3,50 & 0,63 & 2,07 & 4,53 \\
\hline & 3 año & 3,60 & 0,48 & 2,53 & 4,53 \\
\hline & 5 año & 3,78 & 0,64 & 2,47 & 4,73 \\
\hline & Total & 3,61 & 0,59 & 2,07 & 4,73 \\
\hline
\end{tabular}


Diferencias en la mentalización según año de la carrera

De acuerdo con la prueba Kruskal-Wallis no se evidenciaron diferencias significativas entre los grupos estudiantes de primero $(M$ $=3,00 ; D E=1,00)$, tercero $(M=3,12 ; D E=$ $0,71)$ y quinto $(M=3,45 ; D E=0,87)$ año de la carrera para las dimensiones de la escala MentS.

En cuanto a la escala MZQ, no se encontraron diferencias significativas en MZQ total $\mathrm{H}(2)=4,67 ; p=0,09$; MZQ rechazo función flexiva $\mathrm{H}(2)=1,92 ; p=$ 0,38 ; MZQ conciencia emocional $\mathrm{H}(2)=$ 1,$43 ; p=0,48$.

Mientras que sí se observaron diferencias significativas entre los grupos de alumnos de primero, tercero y quinto año en las dimensiones MZQ equivalencia psíquica $\mathrm{H}(2)=6,09 ; p=0,05$; Rango Medio $1=61,08$, Rango Medio $2=63,30$, Rango Medio 3=79,84; $\mathrm{M} 1=3, \mathrm{M} 2=3,16, \mathrm{M} 3=$ 3,$45 ; \operatorname{Md} 1=3,25, \operatorname{Md} 2=3, \operatorname{Md} 3=3,5$ y la dimensión MZQ regulación de afecto $\mathrm{H}(2)$ $=7,87 ; p=0,02$; Rango Medio $1=55,39$, Rango Medio $2=72,17$, Rango Medio $3=$ 76,$27 ; \mathrm{M} 1=3,33, \mathrm{M} 2=3,7, \mathrm{M} 3=3,78 ; \mathrm{Md} 1=$ $3,33, \mathrm{Md} 2=4, \mathrm{Md} 3=4$.

Esto indicaría que los dos aspectos de la mentalización que mejoran en función del avance educacional son la equivalencia psíquica, o sea, lo que se refiere a, en qué medida los estados mentales internos se asimilan y se equiparan con la realidad externa y la regulación afectiva, que alude a la capacidad de modular los afectos.

Como información adicional, se preguntó a los estudiantes, si en algún momento de su vida habían recibido tratamiento psicoterapéutico o psiquiátrico. Del $65,9 \%$ que contestó afirmativamente, el
41,4\% correspondió a psicoterapia. Además, el $55,6 \%$ afirmó que actualmente está haciendo psicoterapia.

\section{Discusión y Conclusiones}

La investigación en psicoterapia en los últimos años, ha mostrado interés en la mentalización como un factor relevante asociado con la efectividad clínica. Además, se ha demostrado que es posible realizar pequeñas mejoras, aunque significativas en la capacidad de mentalización de los terapeutas nóveles (Ensink et al., 2013). En este sentido esta investigación es una primera aproximación al estado actual de la mentalización de futuros psicoterapeutas formados en la UCA.

Otros estudios a nivel general han mostrado que los alumnos de la carrera de psicología presentan mejores habilidades de mentalización que los reportados para la población general (Anupama et al., 2018).

Este estudio proporciona datos relevantes sobre la comparación de las habilidades de mentalización en estudiantes de psicología de grado. Si bien las habilidades de mentalización son mejores en los años más avanzados de sus estudios, estas diferencias sólo son significativas en el caso de la equivalencia psíquica y la regulación del afecto.

La equivalencia psíquica entendida como una modalidad de mentalización ineficaz, asimila los estados mentales internos a la realidad externa, posibilitando que el imaginar una situación crítica pueda generar las mismas emociones que experimentarlo en la realidad. La regulación afectiva se ve imposibilitada ante la inhabilidad de regular el afecto. Esta incapacidad puede dar lugar 
a sentimientos de impotencia y a que las personas se sientan amenazadas por sus propios sentimientos (Hausberg et al., 2012).

Los resultados obtenidos ameritan pensar que la modificación de los puntajes de las dimensiones equivalencia psíquica y regulación del afecto, ambas ligadas al self, introducen la hipótesis de las variables intervinientes del mundo académico que las promueven - programa de prácticas profesionales, contenidos curriculares y/o su integración-, coincidiendo con Lambert (2013), en que son necesarios estudios que permitan especificar las mismas.

Es de sumo interés recalcar que los resultados con relación a la dimensión otros no obtuvieron diferencias significativas a lo largo de la carrera. Dado que el tratamiento psicoterapéutico y su efectividad están ligados a la cualidad de las relaciones con el otro, es importante tomar conciencia de la necesidad de su desarrollo durante la carrera y en los inicios de la profesión. Más aún cuando los resultados en relación con la dimensión de la motivación para la mentalización no se incrementan a lo largo de la carrera.

¿Cómo sería posible saber si un terapeuta está debidamente capacitado? No hay evidencia concluyente en investigaciones previas para responder. Esta pregunta sigue abierta y constituye una línea de interés para futuros estudios. Es de destacar que el poder avanzar en esta respuesta, resulta especialmente importante en países donde un título de grado habilita para hacer psicoterapia, como lo es en Argentina y, por lo tanto, los psicólogos no necesitan completar una especialización para tratar pacientes.

Respecto a las limitaciones del estudio, es importante considerar que éste se ha realizado sólo con estudiantes de psicología de la Pontificia Universidad Católica Argentina, sede Buenos Aires. Una segunda limitación es que los instrumentos no han sido validados aún en la población argentina.

Con relación a futuros estudios, la validación de estos instrumentos sería relevante.

A su vez, resultaría de interés poder estudiar la capacidad de mentalización en estudiantes de otras carreras de grado que se instituyan sobre vínculos terapéuticos, así como en terapeutas recientemente graduados y en los profesionales y agentes de la salud.

El presente estudio representa un aporte significativo, ya que permite plantear la utilidad del entrenamiento en mentalización como parte de la formación de grado de psicología.

\section{Referencias}

Allen, J. G. (2003). Mentalizing. Bulletin of the Menninger Clinic, 67(2: Special Issue), 91-112. https://doi. org/10.1521/bumc.67.2.91.23440

Anupama, V., Bhola, P., Thirthalli, J., \& Mehta, U. M. (2018). Pattern of social cognition deficits in individuals with borderline personality disorder. Asian journal of psychiatry, 33, 105-112. https:// doi.org/10.1016/j.ajp.2018.03.010 Asociación Médica Mundial. (2017). Declaración de Helsinki de la AMM - Principios éticos 
para las investigaciones médicas en seres humanos. ANMAT. http:// www.anmat.gov.ar/comunicados/ HELSINSKI_2013.pd

Bateman, A., \& Fonagy, P. (2008). 8-year follow-up of patients treated for borderline personality disorder: mentalization-based treatment versus treatment as usual. American Journal of Psychiatry, 165(5), 631638.

Bateman, A., \& Fonagy, P. (2013). Mentalization-based treatment. Psychoanalytic Inquiry, 33(6), 595613. https://doi.org/10.1080/07351 $\underline{690.2013 .835170}$

Bateman, A., \& Fonagy, P. (2016). Tratamiento basado en la mentalización para trastornos de la personalidad. España.

Bateman, A. \& Fonagy, P. (2019). Chapter 1: Introduction. En Handbook of Mentalizing in Mental Health Practice (2nd ed., pp. 3-20). American Psychiatric Association Publishing.

Bowlby, J. (1989). Aplicaciones clínicas de una teoría del apego. Ibérica. Paidós.

Bressi, C., Fronza, S., Minacapelli, E., Nocito, E. P., Dipasquale, E., Magri, L., Lionetti, F. \& Barone, L. (2017). Short-term psychodynamic psychotherapy with mentalizationbased techniques in major depressive disorder patients: Relationship among alexithymia, reflective functioning, and outcome variables-A pilot study. Psychology and Psychotherapy: Theory, Research and Practice, 90(3), 299-
313.

Dimitrijević, A., Hanak, N., Altaras Dimitrijević,A.,\& Jolić Marjanović, Z. (2018). The Mentalization Scale (MentS): A self-report measure for the assessment of mentalizing capacity. Journal of personality assessment, 100(3), 268-280.

Ensink, K., Maheux, J., Normandin, L., Sabourin, S., Diguer, L., Berthelot, N., \& Parent, K. (2013). The impact of mentalization training on the reflective function of novice therapists: A randomized controlled trial. Psychotherapy Research, 23(5), 526-538. https://doi.org/10.1 $\underline{080 / 10503307.2013 .800950}$

Epstein, R. M., \& Hundert, E. M. (2002). Defining and assessing professional competence. Jama, 287(2), 226235.

Esposito, G., Passeggia, R., Pepicelli, G., Cannata, A., Parlato F., Freda, M. F. (2020). Mentalizing the university experience: an exploratory study on the relationship between university students' reflective functioning, psychological wellbeing and academic performance. Mediterranean Journal of Clinical Psychology, 8(2). https://doi. org/10.6092/2282-1619/mjcp-2415

Fierro, C., Álvarez, J. F., \& Manzo, G. A. (2018). A Century of Psychotherapy in Argentina: Clinical Psychology, Psychoanalysis and Recent Developments. Revista de Psicología, 27(2), 1-27. http:// dx.doi.org/10.5354/0719$\underline{0581.2019 .52310}$

Fonagy, P. (2015). The effectiveness of 
psychodynamic psychotherapies: an update. World Psychiatry, 14(2), 137-150.

Fonagy, P., \& Target, M. (1996). Playing with reality: I. Theory of mind and the normal development of psychic reality. International journal of psycho-analysis, 77, 217-233.

Fonagy, P., \& Target, M. (1997). Attachment and reflective function: Their role in self-organization. Dev Psychopathol, 9(4), 679700.https://doi.org/10.1017/ s0954579497001399

Fonagy, P., \& Target, M. (2000). Playing with reality: III. The persistence of dual psychic reality in borderline patients. International Journal of Psycho-Analysis, 81(5), 853-873. https://doi. org/10.1516/0020757001600165

Fonagy, P., \& Target, M. (2002). Early intervention and the development of self-regulation. Psychoanalytic Inquiry, 22(3), 307-335.https://doi. org/10.1080/07351692209348990

Fonagy, P., \& Target, M. (2006). The mentalization-focused approach to self pathology. Journal of personality disorders, 20(6), 544-576.https://doi.org/10.1521/ pedi.2006.20.6.544

Fouad, N., Grus, C., Hatcher, R., Kaslow, N., Hutchings, P., Madson, M., Collins, F. \& Crossman, R. (2009). Competency Benchmarks: A Model for Understanding and Measuring Competence in Professional Psychology Across Training Levels. Training and Education in Professional Psychology. 3. 10.1037/a0015832.

Freda, M. F., Esposito, G., \& Quaranta, T. (2015). Promoting mentalization in clinical psychology at universities: A linguistic analysis of student accounts. Europe's journal of psychology, 11(1), 34. http://doi. org/10.5964/ejop.v11i1.812

Goodman, G. (2013). Is mentalization a common process factor in transference-focused psychotherapy and dialectical behavior therapy sessions?. Journal of Psychotherapy Integration, 23(2), 179. https://doi.org/10.1037/ $\underline{\mathrm{a} 0032354}$

Hausberg, M. C., Schulz, H., Piegler, T., Happach, C. G., Klöpper, M., Brütt, A. L., Sammet, I. \& Andreas, S. (2012). Is a self-rated instrument appropriate to assess mentalization in patients with mental disorders? Development and first validation of the Mentalization Questionnaire (MZQ). Psychotherapy Research, 22(6), 699-709.

Horvath, A. O., Del Re, A. C., Flückiger, C., \& Symonds, D. (2011). Alliance in individual psychotherapy. Psychotherapy, 48(1), 9.

Jakobsen, J. C., Gluud, C., Kongerslev, M., Larsen, K. A., Sørensen, P., Winkel, P., Lange, T., Søgaard, U., \& Simonsen, E. (2014). Thirdwave cognitive therapy versus mentalisation-based treatment for major depressive disorder: a randomised clinical trial. $B M J$ open, 4(8), e004903.

Lambert, MJ. (Ed). (2013) Bergin and Garfield's, Handbook of 
Psychotherapy and Behavioral Change (6ta ed.). Nueva York: John Wiley. Recuperado de: https://allmed.net/pdf/bergin-and-garfields-handbook-of-psychotherapyand- behavior-change/ https://doi. org/10.1007/978-3-211-69499257

Lemma, A., \& Fonagy, P. (2013). Feasibility study of a psychodynamic online group intervention for depression. Psychoanalytic Psychology, 30(3), 367.

Lemma, A., Target, M., \& Fonagy, P. (2011). The development of a brief psychodynamic intervention (Dynamic Interpersonal Therapy) and its application to depression: A pilot study. Psychiatry: Interpersonal \& Biological Processes, 74(1), 41-48.

Luyten, P., Lemma, A. \& Target, M.A. (2019). Depression . In A. Bateman \& P. Fonagy (Eds.), Mentalizing in Mental Health Practice (2nd ed., pp. 387-401). American Psychiatric Association.

Midgley, N., Muller, N., Malberg, N., \& Ensink, K. (2019). Children. In A. Bateman \& P. Fonagy (Eds.), Mentalizing in Mental Health Practice (2nd ed., pp. 247-263). American Psychiatric Association.

Orlinsky, D., Ambühl, H., Rønnestad, M., Davis, J., Gerin, P., Davis, M., Willutzki, U., Botermans, J.-F., Dazord, A., Cierpka, M., Aapro, N., Buchheim, P., Bae, S., Davidson, C., Friis-Jorgensen, E., Joo, E., Kalmykova, E., Meyerberg, J., Northcut, T., ... Aapro, N. (1999).
Development of psychotherapists: Concepts, questions, and methods of a collaborative international study. Psychotherapy Research, 9(2), 127-153.

Sharp, C. (2019). Borderline Personality Pathology in Adolescence. In A. Bateman \& P. Fonagy (Eds.), Mentalizing in Mental Health Practice (2nd ed., pp. 281-300). American Psychiatric Association.

Stiles, W. B., Honos-Webb, L., \& Surko, M. (1998). Responsiveness in psychotherapy. Clinical psychology: Science and practice, $5(4), \quad 439-458 . \quad$ https://doi. org/10.1111/j.1468-2850.1998. tb00166.x

Swan, P. \& Riley, P. (2015). Social connection: empathy and mentalization for teachers, Pastoral Care in Education, DOI: 10.1080/02643944.2015.1094120

Target, M., \& Fonagy, P. (1996). Playing with reality: II. The development of psychic reality from a theoretical perspective. International Journal of Psycho-Analysis, 77, 459-479.

Wampold, B. E. (2001). Contextualizing psychotherapy as a healing practice: Culture, history, and methods. Applied and Preventive Psychology, 10(2), 69-86.

Ward, V., House, A., \& Hamer, S. (2009). Developing a framework for transferring knowledge into action: a thematic analysis of the literature. Journal of health services research \& policy, 14(3), 156-164.https:// doi.org/10.1258/jhsrp.2009.008120 


\section{Apéndice A}

\section{Protocolo Datos Demográficos}

Datos Demográficos

1. Sexo: Femenino Masculino

2. Edad:

3. Carrera: Psicología Medicina

4. Año de la cursada de la carrera:

1ro

2 do

3ro

4 to

5to

5. ¿Cuenta con otros estudios académicos?

$\mathrm{Si}$ (especificar)

No

B- En el caso de que haya contestado de manera afirmativa. ¿Cree que este estudio afecto su capacidad de comprender a uno mismo u a otros?

$\mathrm{Si}$

No

6. ¿Alguna vez ha realizado un proceso de psicoterapia o psiquiátrico?

Si (especificar cual)

No

B- En el caso de que haya contestado de manera afirmativa. ¿Está realizando un tratamiento psicológico/psiquiátrico actualmente o ya ha finalizado?

Actualmente

Meses u años desde su finalización 


\section{Apéndice B}

Mentalization Questionnaire [MZQ] (Hausberg et al., 2012)

A continuación encontrará varias declaraciones sobre su capacidad para percibir sus propios sentimientos y los sentimientos de los demás. Nos gustaría que califique cuan de acuerdo esta con estas declaraciones. Responda a cada declaración en la escala de "No estoy de acuerdo" a "Estoy de acuerdo".

\begin{tabular}{|c|c|c|c|c|c|}
\hline Declaración & $\begin{array}{l}\text { No de } \\
\text { acuerdo }\end{array}$ & $\begin{array}{l}\text { Parcialmente } \\
\text { desacuerdo }\end{array}$ & $\begin{array}{l}\text { No estoy } \\
\text { decidido }\end{array}$ & $\begin{array}{l}\text { Parcialmente } \\
\text { de acuerdo }\end{array}$ & $\begin{array}{c}\text { De } \\
\text { acuerdo }\end{array}$ \\
\hline $\begin{array}{l}\text { 1. Si creo que voy a ser criticado } \\
\text { u ofendido, mi miedo aumenta. }\end{array}$ & 1 & 2 & 3 & 4 & 5 \\
\hline $\begin{array}{l}\text { 2. Las explicaciones que me dan } \\
\text { otros, poco ayudan a comprender } \\
\text { mis sentimientos. }\end{array}$ & 1 & 2 & 3 & 4 & 5 \\
\hline $\begin{array}{l}\text { 3. A veces las emociones son } \\
\text { peligrosas para mí. }\end{array}$ & 1 & 2 & 3 & 4 & 5 \\
\hline $\begin{array}{l}\text { 4. Solo creo que alguien me } \\
\text { quiere o está interesado en mí si } \\
\text { tengo pruebas concretas (como } \\
\text { una cita, abrazo o regalo). }\end{array}$ & 1 & 2 & 3 & 4 & 5 \\
\hline $\begin{array}{l}\text { 5. La mayor parte del tiempo es } \\
\text { mejor no sentir nada. }\end{array}$ & 1 & 2 & 3 & 4 & 5 \\
\hline $\begin{array}{l}\text { 6. Muchas veces no puedo } \\
\text { controlar mis sentimientos. }\end{array}$ & 1 & 2 & 3 & 4 & 5 \\
\hline $\begin{array}{l}\text { 7. Es difícil para mí creer que las } \\
\text { relaciones pueden cambiar. }\end{array}$ & 1 & 2 & 3 & 4 & 5 \\
\hline $\begin{array}{l}\text { 8. Tiendo a ignorar sensaciones } \\
\text { físicas de tensión o incomodidad } \\
\text { hasta el punto que ya no lo } \\
\text { puedo ignorar. }\end{array}$ & 1 & 2 & 3 & 4 & 5 \\
\hline $\begin{array}{l}\text { 9. Hablar de mis sentimientos } \\
\text { implica que estos se vuelvan mas } \\
\text { poderosos }\end{array}$ & 1 & 2 & 3 & 4 & 5 \\
\hline $\begin{array}{l}\text { 10. A veces solo me doy cuenta } \\
\text { de mis sentimientos de una } \\
\text { manera } \\
\text { retrospectiva. }\end{array}$ & 1 & 2 & 3 & 4 & 5 \\
\hline
\end{tabular}

Continua en página 79

Revista de Psicología. Año 2021. Vol. 17, No 34, pp. 62-82 
Continua en página 78

\section{Declaración}

No de

Parcialmente
desacuerdo

No estoy

Parcialmente

De

acuerdo

decidido de acuerdo

acuerdo

11. Con frecuencia, es

1

2

3

4

5

difícil para mí percibir mis

sentimientos en su máxima

intensidad.

12. A menudo, me siento amenazado por la idea de que alguien me puede criticar $\mathrm{u}$ ofender.

13. Si alguien bosteza en mi presencia, eso significa que se aburre conmigo.

14. La mayoría de las veces no tengo ganas de hablar sobre mis pensamientos y

sentimientos con otros.

15. A menudo, ni siquiera sé lo que pasa adentro de mí. 
80 Mentalización en estudiantes universitarios de psicología

\section{Apéndice C}

Mentalization Scale [MentS] (Dimitrijevic et al., 2018)

Este cuestionario consiste de 28 ítems. Por favor, lee cada uno de los ítems cuidadosamente y marca un número del 1 al 5, dependiendo de cuan correcto es ese ítem según tu personalidad.

\begin{tabular}{lcccc}
\hline Items & $\begin{array}{c}\text { Completamente } \\
\text { incorrecto }\end{array}$ & $\begin{array}{c}\text { En mayor } \\
\text { parte } \\
\text { incorrecto }\end{array}$ & $\begin{array}{c}\text { Correcto e } \\
\text { incorrecto }\end{array}$ & $\begin{array}{c}\text { En mayor Completamente } \\
\text { parte correcto } \\
\text { correcto }\end{array}$ \\
\hline
\end{tabular}

1. Me parece importante comprender

$\begin{array}{lllll}1 & 2 & 3 & 4 & 5\end{array}$

las razones de mi comportamiento.

2. Cuando saco conclusiones sobre lapersonalidad de otros, observo

5
detenidamente lo que hacen y dicen.

3. Puedo reconocer los sentimientos deotros.

4. A menudo pienso sobre otras

$\begin{array}{lllll}1 & 2 & 3 & 4 & 5\end{array}$

personas y su comportamiento.

5. Usualmente puedo reconocer lo queincomoda a la gente.

6. Puedo simpatizar con los sentimientos de otros.

7. Cuando alguien me molesta

2

23

4

5

intento entender porque reacciono de esamanera.

8. Cuando me siento mal no estoy seguro si es porque estoy triste, enojado o con miedo.

9. No me gusta perder tiempo intentando comprender en detalle el comportamiento de otros.

10. Puedo hacer buenas predicciones sobre el comportamiento de otros cuando conozco cuales son sus creencias y sentimientos.

11. A menudo no puedo explicar,

1

2

3

4

5 inclusoa mí mismo, porque hice algo. 
Continua en página 80

\begin{tabular}{ccccc}
\hline Items & $\begin{array}{c}\text { Completamente } \\
\text { incorrecto }\end{array}$ & $\begin{array}{c}\text { En mayor } \\
\text { parte } \\
\text { incorrecto }\end{array}$ & $\begin{array}{c}\text { Correcto e } \\
\text { incorrecto }\end{array}$ & $\begin{array}{c}\text { En mayor Completamente } \\
\text { parte } \\
\text { correcto }\end{array}$ \\
\hline
\end{tabular}

12. A veces puedo entender los

1

2

3

4

5

sentimientos de alguien antes queme diga algo.

13. Me parece importante

1

2

3

4

5

comprender que pasa en mis

relaciones con laspersonas cercanas

a mí.

14. No quiero enterarme algo de mi mismo que no me vaya a gustar.

1

2

3

4

5

15. Para comprender el

1

2

3

4

5

comportamientode otro necesitamos conocer sus pensamientos, deseos y sentimientos.

16. A menudo hablo de emociones conpersonas cercanas a mí.

17. Me gusta leer libros y artículos dediario sobre temas psicológicos.

1

2

3

4

5

18. Me cuesta admitirme que me sientotriste, lastimado o con miedo.

19. No me gusta pensar en mis problemas.

1

2

3

4

5

sientoriste, lastimado o con miedo.

12

2

3

4

5

20. Puedo describir rasgos

1

2

3

4

5 importantesde personas cercanas a mí con precisión y en detalle.

Continua en página 82 
82 Mentalización en estudiantes universitarios de psicología

Continua en página 81

\begin{tabular}{ccccc}
\hline Items & $\begin{array}{c}\text { Completamente } \\
\text { incorrecto }\end{array}$ & $\begin{array}{c}\text { En mayor } \\
\text { parte } \\
\text { incorrecto }\end{array}$ & $\begin{array}{c}\text { Correcto e } \\
\text { incorrecto }\end{array}$ & $\begin{array}{c}\text { En mayor Completamente } \\
\text { parte correcto } \\
\text { correcto }\end{array}$ \\
\hline
\end{tabular}

21. A menudo estoy confundido

1

2

4

5

acercade mis sentimientos exactos.

22. Es difícil para mi encontrar las

1

2

3

4

5

palabras adecuadas para expresar lo

que estoy sintiendo.

23. La gente me dice que los

1

2

3

4

5

entiendo yque doy buenos consejos.

24. Siempre me ha interesado el

1

2

3

4

5

porquela gente se comporta de cierta manera.

25. Puedo describir con facilidad comome siento.

26. Cuando las personas

hablan sobre sus sentimientos y

necesidades, me

distraigo.

27. Ya que todos dependemos de las

1

2

3

4

5

circunstancias de la vida, no tiene

sentido pensar en las intenciones o

deseos de los demás.

28. Una de las cosas más

1

2

3

4

5

importantes que los niños

deberían aprender es aexpresar sus

sentimientos y deseos. 